\title{
Magnetic Resonance Lymphangiography for the Study of Lymphatic System in Lymphedema
}

\author{
NingFei Liu, MD, $\mathrm{PhD}^{1} \quad$ Yixin Zhang, $\mathrm{MD}, \mathrm{PhD}^{1}$ \\ ${ }^{1}$ Department of Plastic and Reconstructive Surgery, Shanghai 9th \\ People's Hospital, Shanghai Jiao Tong University School of Medicine, \\ Shanghai, China

\begin{abstract}
Address for correspondence NingFei Liu, MD, PhD, Department of Plastic and Reconstructive Surgery, Shanghai 9th People's Hospital, Shanghai Jiao Tong University School of Medicine, 639 Zhi Zao Ju Road, Shanghai 200011, China (e-mail: liuningfei@126.com).
\end{abstract}

J Reconstr Microsurg 2016;32:66-71.

\begin{abstract}
Background Imaging of the lymphatic system is difficult because of its structural and anatomical characteristics, and the conventional diagnostic method, radionuclidebased imaging, has the disadvantage of poor resolution. Magnetic resonance (MR) imaging has been shown the capability of depicting lymphatic channels in lymphedema recently. The purpose of this study was to evaluate the imaging of MR lymphangiography (MRL) in diagnosis of limb lymphedema and its possible role in the microsurgical management of lymphedema.

Methods A total of 710 patients with primary lymphedema $(n=378)$, secondary lymphedema $(n=332)$, were enrolled in the study. Contrast-enhanced lymphangiography was performed with 3.0 T MR unit (Philips Medical Systems, Best, The Netherlands) after intracutaneously injection of gadobenate dimeglumine. Kinetic of enhanced lymph flow within lymphatics and lymph nodes as well as the morphological abnormalities of lymphatic system were evaluated.

Results MRL was able to display the detailed anatomical changes in the vessels and nodes. In primary lymphedema, there are three major types of lymphatic system malformation: (1) only lymph nodes affected, (2) only lymph vessels affected, and (3) both lymph vessels and lymph nodes affected. In secondary lymphedema MRL clearly demonstrated tortuous and dilated collecting lymphatics in lymphedemtous limbs. MRL also provided information concerning the functional status of lymph transport in

Keywords

- lymphedema

- MR lymphangiography

- lymphatic

- lymph node lymphatic vessels and nodes by real-time visualization of enhanced lymph flow in lymphatic channels and within lymph nodes.

Conclusion Contrast MRL was capable of evaluating the anatomical and functional status of lymphatic vessels and lymph nodes in lymphedematous limb. This new imaging shows good potential for use in the diagnosis and surgical management of lymphedema.
\end{abstract}

Impairments of lymph circulation can occur either because of congenital defects leading to malformation of the lymphatic system, or because of secondary damage to lymphatic vessels and lymph nodes. These impairments can cause accumulation of macromolecules and fluid in the tissue, resulting in lymphedema. ${ }^{1}$ Clinical diagnosis of lymphedema in an extremity is typically not difficult, particularly during the

received

April 12, 2014

accepted after revision

June 1, 2014

published online

July 15,2014 late stages of the disease. However, the history and clinical examination do not always indicate the nature or extent of the underlying abnormality (i.e., the anatomical extent, pathology, and cause).

The development of clinical lymphology was slow for a long time. This was largely due to the lack of optimal diagnostic methods, including methods for lymphatic system

Copyright $\odot 2016$ by Thieme Medical Publishers, Inc., 333 Seventh Avenue, New York, NY 10001, USA. Tel: +1(212) 584-4662. 
imaging. The commonly used lymphoscintigraphy, with an isotopic contrast agent, has insufficient resolution to accurately outline the internal anatomy of the lymph nodes and lymphatic vessels. The measurement of tracer clearance at the injection point and accumulation of the tracer in the inguinal lymph nodes may be valuable in functional evaluation but are limited in accuracy. ${ }^{2}$ Direct lymphangiography using iodine oil as the contrast agent, which is capable of visualizing the lymphatics, is no longer routinely performed because it is highly invasive and difficult to perform, and also can lead to life-threatening complications. ${ }^{3}$

Neither of the above methods is able to dynamically visualize lymphatic vessels and lymph nodes. It is, therefore essential to use a combination of imaging modalities that, in addition to mapping the anatomical distribution of the affected lymphatic system, is capable of defining its function. Magnetic resonance imaging (MRI) has recently emerged as a new method for diagnosing lymphostatic diseases. ${ }^{4-7}$ Since 2007 we have adopted three-dimensional (3D) dynamic magnetic resonance lymphangiography (MRL) using the paramagnetic contrast agent Gd-BOPTA (gadolinium benzyloxypropionictetraacetate) to assess the lymphatic system architecture and lymph drainage in extremities affected by lymphedema. ${ }^{8}$ MRL is currently the routine diagnostic modality for lymphedema in the authors' clinic. This article presents the results of MRL in the diagnosis of lymphedema based on the examination of 710 patients for the purpose of leading to a better understanding of this relatively new technique.

\section{Materials and Methods}

The present study included 710 patients (335 men, 375 women; mean age 35 years, range: 5-78 years). Among them, 378 were diagnosed with primary extremity lymphedema and 332 with secondary lymphedema after lymph node dissection, surgery and/or radiation therapy, lymph node metastasis, injury or lymphadenitis. The mean duration of disease across all patients was 8 years, and ranged from 1 month to 55 years.

MRL was performed as described previously ${ }^{8}$ with a $3.0 \mathrm{~T}$ MR unit (Philips Medical Systems, Best, The Netherlands). First, 3D heavily T2-weighted MRI was performed. After intradermal injection of Gd-BOPTA into the first and second digital web space of bilateral hand/feet (0.7-0.8 mL per point; MultiHance; Bracco, Milan, Italy), 3D fast spoiled gradientrecalled echo T1-weighted images were acquired with a fat saturation technique at consecutive time points for dynamic observation. Images were reconstructed using maximum intensity projection to map the lymphatic vessels. In addition to mapping lymphatic distribution patterns, the lymph flow speed in lymphatic collectors was recorded to calculate the length of the enhancement of the lymphatic vessels per minute of testing time using the formula:

$$
\begin{aligned}
\text { Speed }(\mathrm{cm} / \mathrm{min})= & \text { Total length of visualized lymph } \\
& \text { vessel }(\mathrm{cm}) \div \text { Inspection time }(\mathrm{min}) .
\end{aligned}
$$

Images of regional lymph nodes were acquired before and after injection of contrast agent. The contrast enhancement in regional lymph nodes was recorded and measured as the node to muscle signal intensity ratio for direct comparison between bilateral inguinal lymph nodes.

\section{Results}

\section{Imaging of the Lymphatic System in Primary Lymphedema}

After intracutaneous injection, the contrast agent was quickly taken up by the primary lymph vessels and transported with the lymph. The enhancement of lymphatic vessels and lymph nodes was inspected accordingly during the dynamic MRL. The drainage patterns of lymphatic vessels and nodes were clearly demonstrated. In most contralateral healthy limbs no enhanced or only a few light lymph vessels were visualized (-Fig. 1a), and the inguinal nodes enhanced quickly and completely ( - Fig. 1b). The changes in the lymphatic system in primary lymphedema may occur in the lymph vessels, in the nodes, or in both. The number of lymphatic vessels was from none or one to many, and the diameter was from 0.5 to $8 \mathrm{~mm}$ in lymphedematous limbs.

The lymphatic abnormalities fell into two major categories: aplasia/hypoplasia or hyperplasia. In aplasia/hypoplasia the peripheral vessels could be absent or severely hypoplastic in number and size. In hyperplasia, varicose lymphatic vessels that were numerous, tortuous, and dilated were found in the limb and trunk (- Fig. 1a). Common pathological features of the affected lymph nodes included: significant enlargement, smaller in size and number, absent, irregular in outline, heterogeneous in texture, and partial (marginal or central region) contrast enhancement (-Fig. 1b). Combining the lymphatic and lymph node anomalies seen on MRL, the malformation of the lymph system in primary lymphedema included three major types:

1. Only lymph nodes affected with abnormal nodal structural (17\%)

2. Only lymph vessels affected (32\%) with three subtypes (lymphatic aplasia, hypoplasia, and hyperplasia)

3. Both lymph vessels and lymph nodes affected (51\%)

The speed of enhanced lymph flow ranged from 0.301 to $1.48 \mathrm{~cm} / \mathrm{min}$ (-Fig. 2a). The speed of lymph transport seems largely individual in primary lymphedema. However, the transport of lymph was significantly delayed in varicose lymphatic vessels compared with that in the contralateral nonedematous limb. The contrast-enhanced MR images showed marked asymmetrical accumulation of contrast agent between the nodes of the edematous limb and contralateral limb of patients with unilateral lymphedema (-Fig. 2 b), and the contrast enhancement measured as the node to muscle signal intensity ratio in lymphedematous limbs was significantly lower than in the contralateral limbs.

\section{Imaging of the Lymphatic System in Secondary Lymphedema}

In acute secondary lymphedema, for example, resulting from a tumor in the inguinal lymph node, the afferent lymphatic vessel might be extremely dilated (diameter $>1$ 


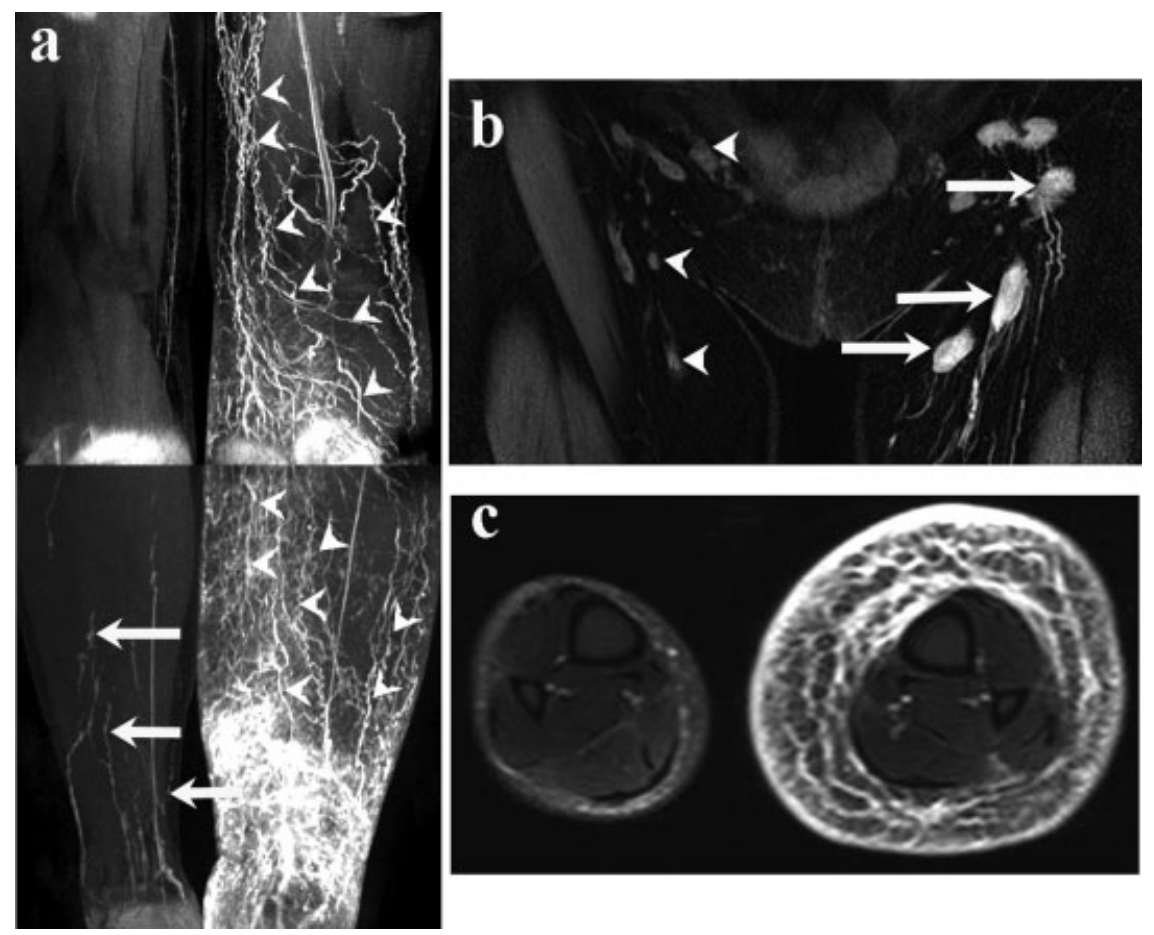

Fig. 1 (a) A 36-year-old woman with primary lymphedema of left lower limb, numerous tortuous and dilated lymph vessels (arrowheads) were demonstrated in contrast with contralateral no edema right limb with a few light lymphatic vessels (arrows) on postcontrast MRL imaging. (b) Enlarged inguinal lymph node with heterogeneous appearance and inhomogeneous enhancement in edema limb (arrows) in contrast with the contralateral nodes that were evenly enhanced (arrowheads). (c) Axial section of T2-weighted MR image shows the thickness and edema of the subcutis (high signal intensity) of the left lower limb. MRL, magnetic resonance lymphangiography.

$\mathrm{cm}$ ) and/or even disrupted ( - Fig. 3a). Tortuous and dilated collecting lymphatics were also clearly seen in the lymphedematous arms after mastectomy in contrast with a single lymphatic in the contralateral no edema arm (-Fig. 3b). Dilated lymph collectors were seen in the distal third of the lower leg without visualization of proximal channels in a considerable number of patients ( - Fig. $\mathbf{3 c}$ ), which indicated obliteration of the lymphatic channels in the proximal part of the limb.

In patients with secondary lymphedema of a lower extremity after surgery and/or radiation, the inguinal lymph nodes were found to be smaller, decreased in number, irregular in shape, and with poor contrast enhancement. In patients with lymph node metastasis, the inguinal and iliac nodes were found to be enlarged and partial or totally without contrast enhancement.

In general, edema was more extensive and tissue fibrosis progressed during the course of the disease in both types of lymphedema. On cross-sectional MRI it was possible to localize the water and observe other pathological changes such as fat deposition in the subcutaneous layer (-Fig. 1c), and it was therefore possible to recognize the disease stage in affected limbs.

\section{Discussion}

Although, all lymphostatic diseases result in lymphedema, the pathogenesis of lymphatic disorders is quite complicated.
The anatomical changes in the lymphatic system (lymphatic vessels and lymph nodes) are closely related to its function, so that localization of the involved parts and characterization and functional analysis of the interrupted lymph flow are equally valuable in the diagnosis of lymphatic circulation disorders.

In this clinical study, MRL with Gd-BOPTA as a contrast agent quickly and sufficiently visualized the lymphatic pathway and lymph nodes draining from the intracutaneous injection sites in lymphedematous limbs, and enabled both morphological and functional assessments of the lymphatic system. Contrast-enhanced MR images specifically depicted the lymphatic vessels in the limb with disturbance of lymph back flow but not the lymphatic vessels in healthy limbs. Contrast-enhanced lymphatic channels in affected limbs were visualized together with accumulation of edema fluid in the tissue. ${ }^{9}$

Therefore, a lymph circulation disorder should be highly suspected when contrast-enhanced lymphatic vessels are visualized with this test. The specificity of the absorption and transportation of the contrast agent by the lymphatic system made possible visualization of the finely detailed morphological changes in lymphatic vessels as well as regional lymph nodes by high-resolution MR imaging. MRL clearly depicted lymph vessels with various morphological abnormalities, in both small solitary vessels (1 $\mathrm{mm}$ in diameter), and in networks of tortuous and dilated lymphatic trunks (10 $\mathrm{mm}$ in diameter). The quality of MRL imaging for mapping lymphatic patterns was comparable to that of direct 

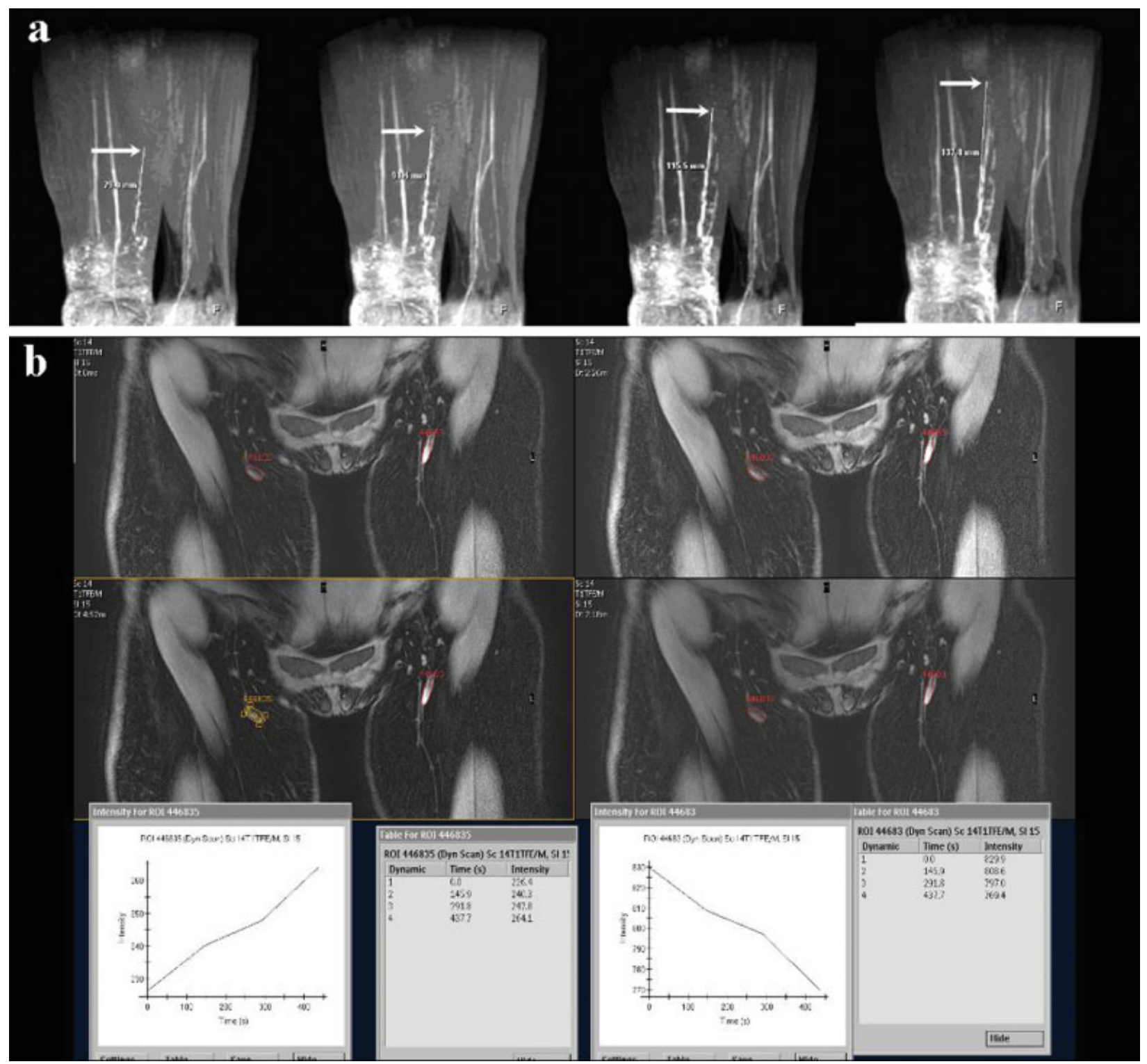

Fig. 2 Dynamic images of enhanced lymph flow. (a) Observation of movement of contrast-enhanced flow (arrows) in a lymphedema limb. (b, c) Coronal postcontrast images of real-time record of comparison of nodal enhancement in edematous (right) and nonedematous (left) limbs in a series images. The wash-in curve was prolonged in the affected limb in contrast with quick wash-out curve in unaffected limb.

lymphangiography with the use of an iodine oil contrast agent.

The detection rate of lymphatic vessels and lymph nodes with MRL was much higher than that found with lymphoscintigraphy. ${ }^{10}$ MRL imaging was able to display distinct morphological changes in regional lymph nodes, and more structural abnormalities could be displayed by evaluating intranodal contrast-filling patterns. Intranodal pathology was indicated by a heterogeneous appearance, partial nodal enhancement, or irregularity in nodal outline, which could be the distinct characteristics in some types of primary ${ }^{8,11}$ and secondary lymphedema. ${ }^{12}$

Previous diagnostic approaches such as direct lymphangiography and lymphoscintigraphy may indicate the functional status of the lymphatic system and allow assessment of patency, but none of these techniques are capable of real- time lymph flow monitoring. Neither iodized oil contrast agents nor isotopically labeled tracers are able to measure enhanced lymph flow velocities in lymphatic vessels, because of the slow movement and slow absorption of the contrast agent, and the prolonged time (hours or days) involved in imaging. The present study showed that the rapid transportation of contrast agent by draining lymphatic vessels and regional lymph nodes during MRL allows consecutive and real-time evaluation of the transport function of lymphatic vessels and lymph nodes within a reasonable length of time. Tracing the movement of enhanced flow within lymphatic vessels allowed quantitative assessment of abnormal lymph flow kinetics. Comparison of dynamic nodal enhancement between the edematous and contralateral limbs and analysis of the time-signal intensity curves could clarify the delayed or decreased transport of lymph in individual nodes and 

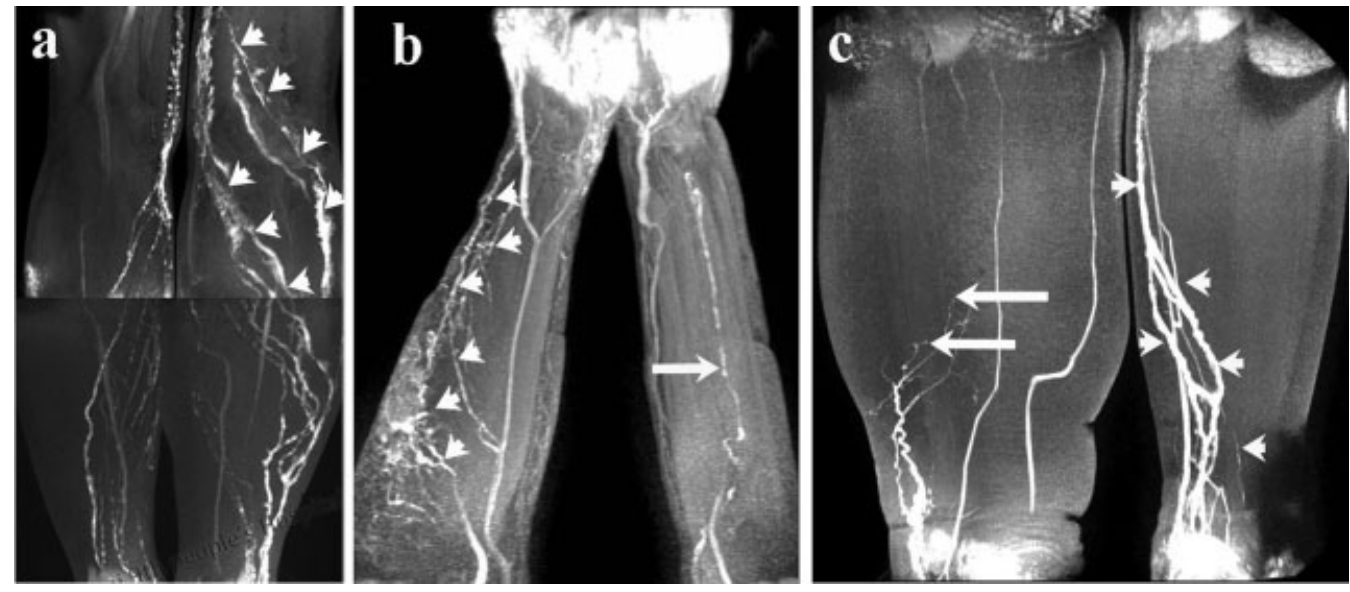

Fig. 3 (a) Postcontrast MR lymphangiogram displays significantly dilated lymphatic vessels (arrowheads) of the left lower limb in a patient with inguinal lymph nodal occupation. (b) Contrast-enhanced lymphatic vessels (arrowheads) of the right upper limb in contrast with single lymphatic (arrow) in the contralateral no edema arm on the MRL imaging in a woman after mastectomy. (c) Contrast-enhanced lymphatics ended at the distal third of the right lower leg (arrows) without visualized proximal channels in a patient with lymphedema after surgical treatment of endometrial carcinoma. Dilated lymphatic collectors were visualized in the left leg (arrowheads). MRL, magnetic resonance lymphangiography.

allow quantitative assessment of abnormal nodal lymph flow kinetics. The combination of lymphatic vessel and lymph node images may then offer an integrated picture of an affected lymphatic system.

Taking together the morphological observation of lymphatic vessels and lymph nodes by MRL imaging made possible the classification of lymphatic anatomical abnormalities in primary lymphedema. ${ }^{11}$ By accurately localizing the damage to the lymphatic system in secondary lymphedema, MRL could help in choosing the appropriate surgical (e.g., selecting a suitable vessel for lymphovenous anastomosis) or conservative treatment, and could also be used during follow-up for the evaluation of outcome after therapy.

Contrast-enhanced 3D MRL not only demonstrated the lymphatic vessels and lymph nodes, but also identified extralymphatic abnormalities such as the location of edema and other pathological changes resulting from stagnant lymph such as fat deposition and tissue fibrosis, and was also able to differentiate lymphedema from other types of edema.

MRI has several potential advantages compared with lymphoscintigraphy, including higher spatial resolution enabling depiction of lymphatic channels, higher temporal resolution, 3D images, and the absence of ionizing radiation. MRL as currently used is safe and repeatable. No side effects have yet been found during or after the examination. It is suitable for diagnosis and the differential diagnosis of various types of chronic peripheral lymphedema, malignant lymphedema, venous edema, lymphovenous edema, lipedema, and other lymphatic vascular diseases. Like routine MRI examination, MRL should not be performed in patients with any electrically, magnetically or mechanically activated implants. The MRL technique still has limitations. Except for extremely dilated vessels in patients with lymphatic hyperplasia, MRL with Gd-BOPTA is unable to depict deep lymphatic system structures such as lumbar trunks, the cistern, and the thoracic duct.
In conclusion, MRL is much more sensitive and accurate for detecting anatomical and functional abnormalities of the lymphatic system in contrast to the previous and currently used diagnostic methods. This new lymphatic system imaging test shows good application prospect in the microsurgical management of lymphedema.

\section{Funding}

Chinese Nature Science Foundation (Grant no. 30772262, 81272146); Shanghai Science and Technology Committee (Grant no. 12401900504).

\section{References}

1 Foldi E, Foldi M. Lymphostatic diseases. In: Foldi M, Foldi E, Kubik S. eds. Textbook of Lymphology. München: Elsevier GmbH; 2003: 231-318

2 Szuba A, Shin WS, Strauss HW, Rockson S. The third circulation: radionuclide lymphoscintigraphy in the evaluation of lymphedema. J Nucl Med 2003;44(1):43-57

3 Silvestri RC, Huseby JS, Rughani I, Thorning D, Culver BH. Respiratory distress syndrome from lymphangiography contrast medium. Am Rev Respir Dis 1980;122(4):543-549

4 Liu N, Wang C, Sun M. Noncontrast three-dimensional magnetic resonance imaging vs lymphoscintigraphy in the evaluation of lymph circulation disorders: A comparative study. J Vasc Surg 2005;41(1):69-75

5 Barrett T, Choyke PL, Kobayashi H. Imaging of the lymphatic system: new horizons. Contrast Media Mol Imaging 2006;1(6): 230-245

6 Liu NF, Lu Q, Wang CG, Zhou JG. Magnetic resonance imaging as a new method to diagnose protein losing enteropathy. Lymphology 2008;41(3):111-115

7 Dayan JH, Dayan E, Kagen A, et al. The use of magnetic resonance angiography in vascularized groin lymph node transfer: an anatomic study. J Reconstr Microsurg 2014;30(1):41-45

8 Liu NF, Lu Q Jiang ZH, Wang CG, Zhou JG. Anatomic and functional evaluation of the lymphatics and lymph nodes in 
diagnosis of lymphatic circulation disorders with contrast magnetic resonance lymphangiography. J Vasc Surg 2009;49(4): 980-987

9 Liu NF, Lu Q Yan ZX. Lymphatic malformation is a common component of Klippel-Trenaunay syndrome. J Vasc Surg 2010; 52(6):1557-1563

10 Liu NF, Lu Q, Liu PA, Wu XF, Wang BS. Comparison of radionuclide lymphoscintigraphy and dynamic magnetic resonance lymphan- giography for investigating extremity lymphoedema. Br J Surg 2010;97(3):359-365

11 Liu NF, Yan ZX, Wu XF. Classification of lymphatic-system malformations in primary lymphoedema based on MR lymphangiography. Eur J Vasc Endovasc Surg 2012;44(3):345-349

12 Liu N, Yan Z, Lu Q, Wang C. Diagnosis of inguinal lymph node metastases using contrast enhanced high resolution MR lymphangiography. Acad Radiol 2013;20(2):218-223 